(C) 2017 IEEE. Personal use of this material is permitted. Permission from IEEE must be obtained for all other uses, in any current or future media, including reprinting/republishing this material for advertising or promotional purposes, creating new collective works, for resale or redistribution to servers or lists, or reuse of any copyrighted component of this work in other works. 


\title{
A Novel Method to Avoid Degradation due to Demagnetization of PM Linear Generators for Oceanic Wave Energy Extraction
}

\author{
Omar Farrok ${ }^{1}$, Md. Rabiul Islam², Senior Member, IEEE, Youguang Guo³, Senior Member, IEEE, and \\ Jianguo Zhu ${ }^{3}$, Senior Member, IEEE
}

\begin{abstract}
${ }^{1}$ Department of Electrical and Electronic Engineering, Ahsanullah University of Science \& Technology, Dhaka-1208, Bangladesh, ${ }^{2}$ Department of Electrical \& Electronic Engineering, Rajshahi University of Engineering \& Technology, Rajshahi-6204, Bangladesh ${ }^{3}$ University of Technology Sydney, New South Wales 2007, Australia,

Email: omarruet@gmail.com, rabiulbd@hotmail.com, ris_eee@ ruet.ac.bd, youguang.guo-1@uts.edu.au, jianguo.zhu@uts.edu.au
\end{abstract}

\begin{abstract}
Demagnetization is one of the noticeable problems in almost every permanent magnet linear generator (PMLG) for oceanic wave energy conversion. To avoid the degradation of electrical power generation capability, a PMLG with a new feature of variable air gap length is proposed in this paper. To verify the effectiveness of the proposed method, a PMLG has been designed which is simulated for both the fixed and variable air gaps. The finite element analysis is performed for simulation for analysis of the PMLG with the conventional and the proposed methods by using the software package ANSYS/Ansoft. The voltage, current, power, magnetic flux density, and force components of the PMLG are analyzed and discussed. The simulation results show that the new method can effectively avoid the demagnetization problem.

Index Terms-Demagnetization, oceanic wave energy conversion, permanent magnet linear generator
\end{abstract}

\section{INTRODUCTION}

Due to the limited traditional energy resources as well as the negative environmental impacts of excessive utilization of fossil fuels, the application of renewable energy resources (RERs) for electrical power generation has been growing rapidly for the last couple of decades [1]. The oceanic wave has a high power density compared to other existing RERs and it is widely available, predictable and environment friendly. Without difficulty, the total harvestable electrical energy from the near coastline is estimated as 2.5 billion $\mathrm{kWh}$, which is $10 \%$ of the global power consumption [2]. Among various wave energy devices, the direct drive system coupled with the permanent magnet linear generator (PMLG) has been proven to be very efficient for oceanic wave energy conversion (OWEC) systems.

A conventional PMLG connected to a point absorber type power take off system is shown in Fig. 1. Almost all of these conventional PMLGs are such designed, so that the stator and translator can maintain a constant air gap length. A number of PMLGs have been studied such as those in [3]-[9] and it is found that all of them have fixed air gap between their stators and translators. The air gap of any particular PMLG is considered fixed in all the way of its stroke length.

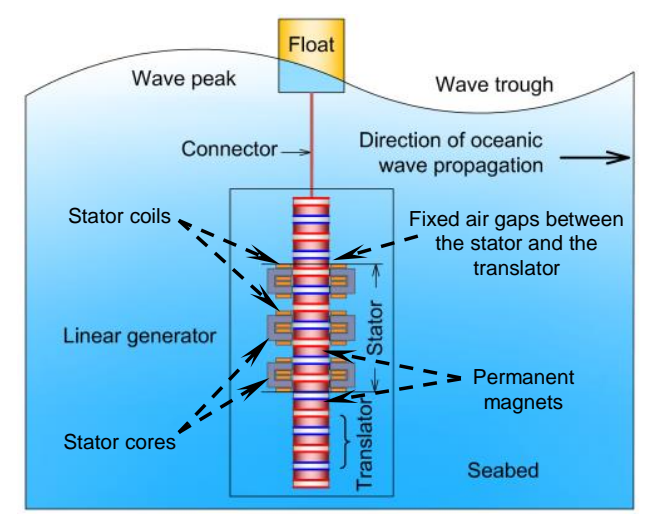

Fig. 1. The conventional linear generator with fixed air gap length.

According to the construction, these PMLGs can be of either tubular [10] or flat type [11]. The traditional PMLGs usually have complex translator structures and suffer from unexpected rise of translator temperature, which often causes mechanical damage and irreversible demagnetization of permanent magnets [11]. As explained in [12], the PMLG with double-sided flat rectangular permanent magnets and direct coupling with a point absorber has certain benefits over other types of structures. A theoretical concept of a new design of the translator of a flat PMLG for OWEC is explained in [13]. A fuzzy logic based improved controller has been designed and simulated in [14] to stabilize the output voltages of a flat type PMLG for the OWEC systems. A shape optimization of the linear generators has been introduced in [15], where the result of using the proposed optimized stator shape of a flat type PMLG is to generate more electrical power using less amounts of steel cores. Various types of PMLGs have been studied in [16] and [17] and it is found that demagnetization occurs in almost every PMLG. To avoid demagnetization, a novel electromagnetic linear generator [16] and a superconducting magnetic flat linear generator [17] have been proposed. However, further studies show that the electromagnetic linear generator suffers from the fact of high copper loss while the superconducting one is expensive to build. 
In this paper, a new approach is proposed to solve the demagnetization problem by adopting variable air gap length between the stator and translator. A preliminary study [18] has shown that when the air gap length varies as a function of the translator velocity, the demagnetization can be avoided and the degradation of PMLGs due to demagnetization can be effectively eliminated so that PMLGs can generate electricity at their full capacity most of the time.

\section{MAthematical Model of the Proposed METHOD}

Fig. 2 depicts the proposed method for two different translator positions with respect to the stator considering the linear wave. When the float touches the wave peak or wave trough, the translator connected to a buoy reaches its end position with a lower velocity as shown in Fig. 2(a). The vertical speed of the translator is almost zero at both ends and maximum at its center position as shown in Fig. 2(b). The relationship among the float, vertical position of the wave surface, and velocity can be visualized from Fig. 3. Therefore, the air gap length decreases when the translator end approaches the stator and vice-versa.

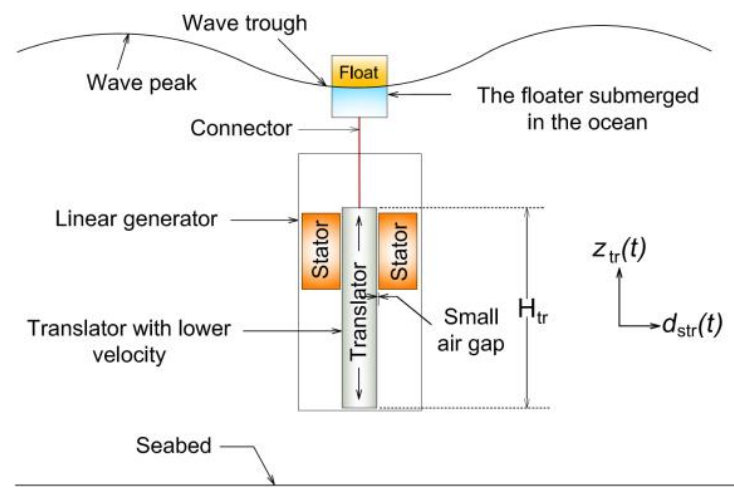

(a)

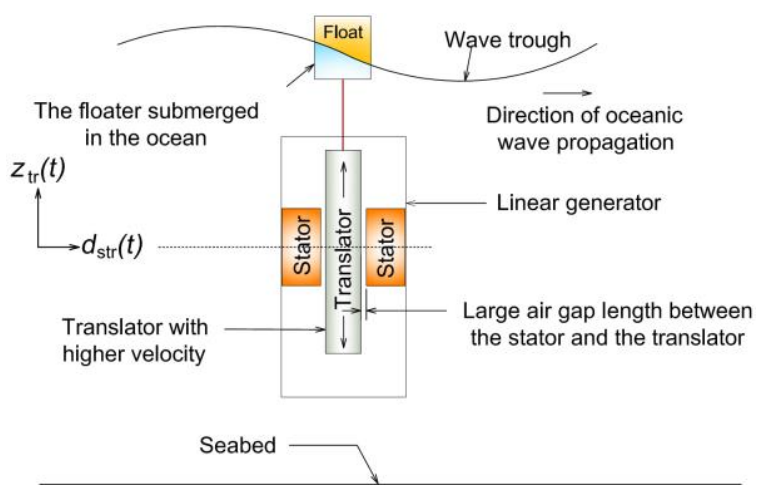

(b)

Fig. 2. Variation of air gap length for different translator velocities. (a) Small air gap is required for lower translator velocity. (b) Large air gap is required for higher translator velocity.

The translator vertical position, $z_{t r}(t)$, and velocity, $v_{t r}(t)$, of the translator are interrelated. The air gap length, $d_{s t r}(t)$, can be represented as a function of position as follows:

$$
\begin{gathered}
d_{s t r}(t)=d_{\mathrm{min}}+d_{\mathrm{var}}\left[1-\frac{\cos \pi\left\{z_{t r}(t)\right\}}{H_{t r}}\right] \\
z_{t r}(t)=\frac{H_{t r}}{2} \sin \left(\frac{2 \pi}{T} t \pm \alpha_{i}\right) \\
v_{t r}(t)=\frac{H_{t r} \pi}{T} \cos \left(\frac{2 \pi}{T} t \pm \beta_{i}\right)
\end{gathered}
$$

where $d_{\text {min }}$ and $d_{v a r}$ represent the minimum and variable air gap lengths, respectively. $H_{t r}, v_{t r}(t)$, and $T$ represent the height, velocity and period of the translator motion, respectively. The initial arbitrary phase angles are denoted by $\alpha_{i}$ and $\beta_{i}$. If, $f\left\{z_{t r}(t)\right\}=\frac{\cos \pi\left\{z_{t r}(t)\right\}}{H_{t r}}$, the flux variation, $\Phi_{s t}$ can be represented as:

$\Phi_{s t}(t)=\vec{\Phi} \sin \left(\frac{2 \pi}{\lambda} z_{t r}(t) \pm \alpha_{i}\right)\left[2 f\left\{z_{t r}(t)\right\}-f^{2}\left\{z_{t r}(t)\right\}\right](4)$

$$
\begin{aligned}
v_{\text {gen }}(t)=\vec{V}_{\max } \cos \left(\frac{2 \pi}{T} t\right) \cos \left\{\frac{2 \pi A_{t r}}{\lambda} \sin \left(\frac{2 \pi}{T} t\right)\right\} \\
{\left[2 f\left\{z_{t r}(t)\right\}-f^{2}\left\{z_{t r}(t)\right\}\right] }
\end{aligned}
$$

\section{MinimiZATION OF DEMAGNETIZATION}

Fig. 3 shows the nature of vertical position and velocity of a translator with respect to time in all PMLGs. The generated voltage, electrical power and hence the armature current is directly proportional to the translator velocity considering the same load where the nature of current and power of the PMLG are shown in Fig. 3. The direction of magnetic flux of the PMLG is given in Fig. 4.

Basically, all permanent magnets are hard magnetic materials which are usually characterized by a wide hysteresis loop of $B-H$ with four quadrants. In fact, while these permanent magnets are performing their operation in PMLGs to produce electricity, the $B-H$ curves are operated in the second quadrant of the $B-H$ loop as illustrated in Fig. 5. Therefore, the basis for the calculation of a permanent magnet is the portion of its hysteresis loop located in the second quadrant, known as the demagnetization curve. Let us consider a new permanent magnet that was not used in any operation, where $B_{r}$ is the magnetic remanence or remanent magnetism and $H_{c}$ is the coercive force. Before starting generator operation the magnetic flux density, $B$ is located at point $a$. When electricity is generated in the armature coil for the first time, due to the induced armature current a reverse magnetic field is created according to the Lenz's law. The induced emf/phase $E_{p}$ can be determined by

$$
E_{p}=\pi \sqrt{2} f N_{1} k_{w l} \varphi_{g}
$$


where $\Phi_{g}$ is the air gap flux which can be expressed as

$$
\varphi_{g}=\varphi_{f}-\varphi_{a}-\varphi_{l}
$$

where $\Phi_{f}, \Phi_{a}$, and $\Phi_{l}$ are the excitation flux, armature reaction flux and leakage flux, respectively, which are shown in Fig. 4. The winding factor is denoted by $k_{w l}$. At no load condition, $\Phi_{g} \approx \Phi_{f}$ as $\Phi_{l}$ is very small.

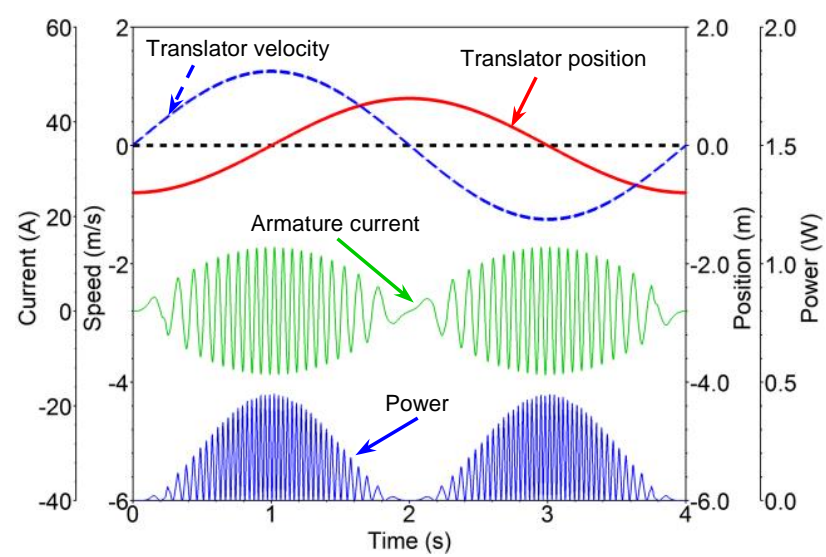

Fig. 3. The winding current and power generation of a PMLG with respect to the translator position and velocity.

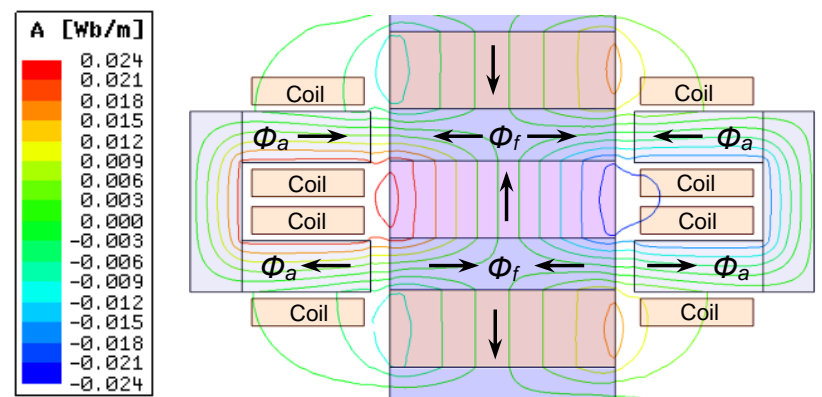

Fig. 4. The demagnetization occurs in permanent magnet of the conventional PMLG.

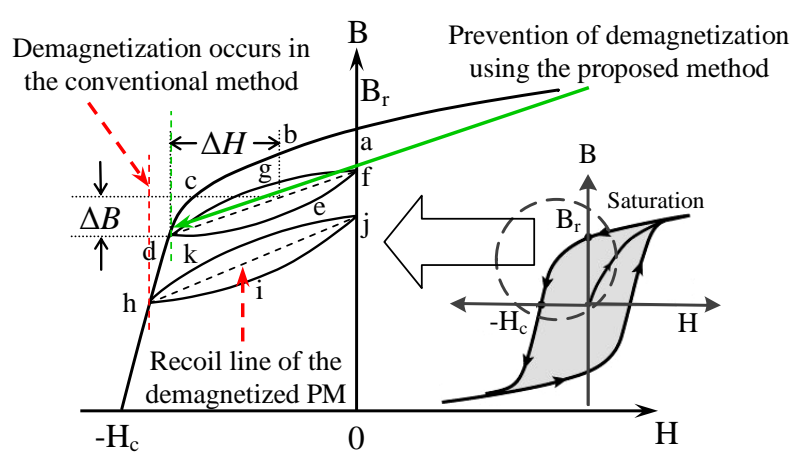

Fig. 5. Demagnetization curve of a permanent magnet (PM).

Therefore, when the generator supplies electrical power to the load, due to armature reaction, $\Phi_{a}$ mainly opposes the excitation flux. This reverse magnetic field is applied to the previously magnetized permanent magnet, and therefore, the magnetic flux density drops down from point $a$ to the magnitude determined by the point $d$ followed by the abcd line. When the PMLG is not running, that is, when it does not produce electricity at that moment when the reverse magnetic field does not exist, the flux density returns to the point $f$ instead of point $a$ followed by the def line according to a minor hysteresis loop. Thus, the application of a reverse field has reduced the remanence. Reapplying an opposite magnetic field intensity will again reduce the flux density, completing the minor hysteresis loop defgd by returning the core to approximately the same value of flux density at the point $d$ as before. The minor hysteresis loop may usually be replaced by a straight line $d f$ for simplification called the recoil line. This line has a slope called the recoil permeability $\mu_{\text {rec }}$. As long as the negative value of applied magnetic field intensity does not exceed the maximum value corresponding to the point $d$, the permanent magnet may be regarded as reasonably permanent. Due to the design strategy, the proposed method restricts applying further negative field by maintaining larger air gap length for higher translator velocity. However, a further greater negative field intensity $H$ is applied without intension in the conventional PMLG because of not having such an arrangement due to the higher translator velocity which depends on the natural motion of the sea wave. In this case, the magnetic flux density will be reduced to a value, say, point $h$ lower than that at point $d$. As the magnitude of reverse magnetic field depends on the velocity of the PMLG for a particular load, which varies with wave motion, therefore, $B$ will be reduced to a new value. On the removal of $H$, a new and lower recoil line $h j$ will be established and again a secondary hysteresis loop hijkh will be formed and will never return to the previous minor hysteresis loop defgd because there is no way in the PMLG to give sufficiently high magnetic field intensity in the positive $H$ direction so that it can re-gain remanent magnetism $B_{r}$.

\section{DESIGN OF THE PMLG FOR ANALYSIS}

The cross sectional view from the front elevation of the PMLG is sketched in Fig. 6(a). It is formed by two major parts, namely, the stator and the translator. The permanent magnets are placed between the translator cores and the armature is situated in the stator cores. Plain copper wires have been considered for windings. Two sets of stator with windings are placed on both the left and the right sides of the translator. Each of the stator contains a winding having two coils that are $180^{\circ}$ electrical phase shifted from each other. Therefore, the total number of coils is four and these are connected in series as shown in Fig. 6(b), which represents the equivalent circuit diagram of the PMLG. In Fig. 6(b), the equivalent series resistance and inductance of these four coils are represented as $R_{\text {windings }}$ and $L_{\text {windings }}$, respectively. The generated voltages from these four coils are marked in a dashed rectangle and represented by $E_{g}$ as their combination.

The construction supports the translator moving vertical direction with respect to stator. The orientation of the permanent magnet array is such that the North and South poles of two consecutive permanent magnets are opposite to each other as labeled in Fig. 6 (a). 


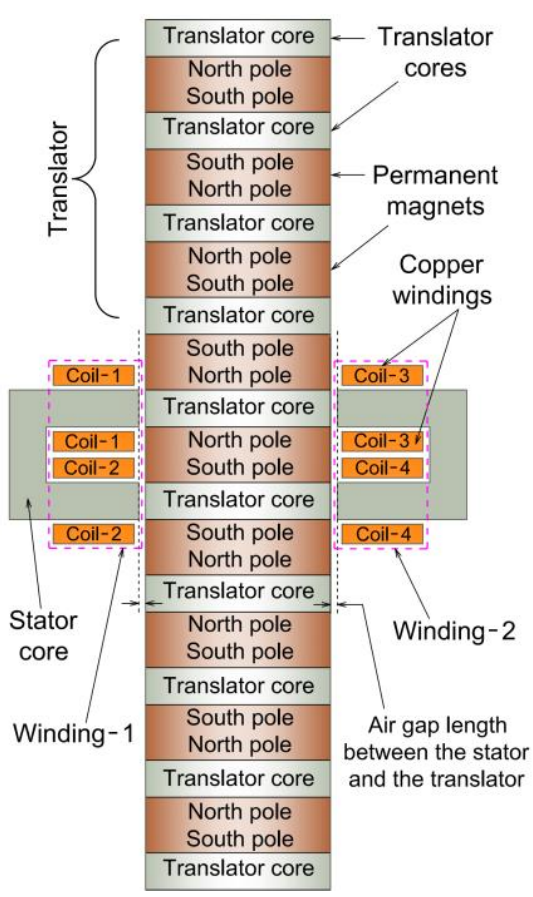

(a)

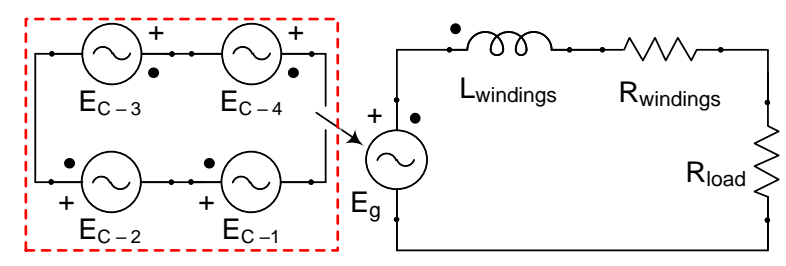

(b)

Fig. 6. (a) Construction of the PMLG and (b) its equivalent circuit diagram.

\section{SIMULATION RESULTS}

The stator winding current waveforms are simulated for the conventional fixed air gap PMLG and the proposed adjustable air gap PMLG, respectively, considering the same design and parameters. The proposed method can limit the armature current to a specified maximum value inherently to prevent demagnetization as shown in Fig. 7. The terminal voltage, load current, and magnetic flux linkage are shown in Fig. 8. The effectiveness of the proposed method has been simulated, and is shown in Fig. 9 for three different limiting currents, namely, $I_{1}(8 \mathrm{~A}), I_{2}$ (10 A), and $\mathrm{I}_{3}(12 \mathrm{~A})$. Fig. 10 depicts the electrical power generation from the same PMLG with and without using the proposed method for comparison maintaining the same maximum current rating.

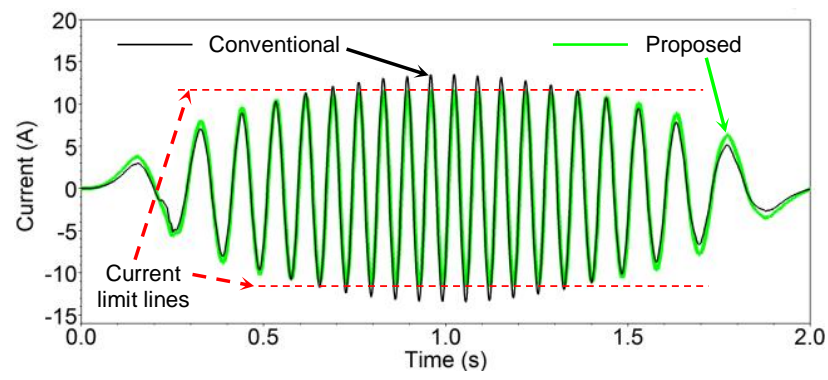

Fig. 7. The armature current for a specific load.

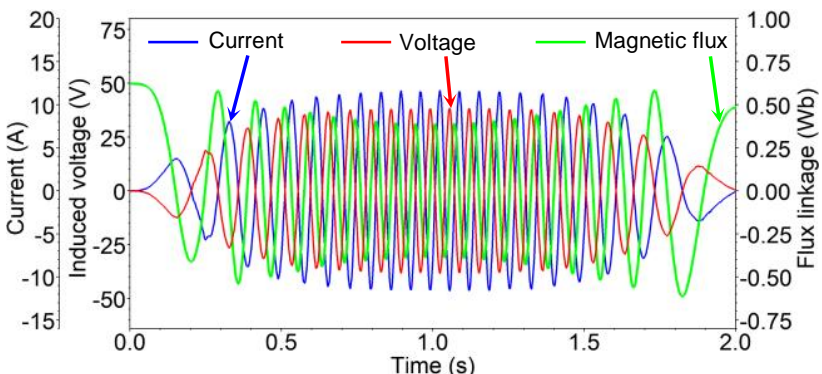

Fig. 8. The voltage, current, and magnetic flux for one cycle of oceanic wave.

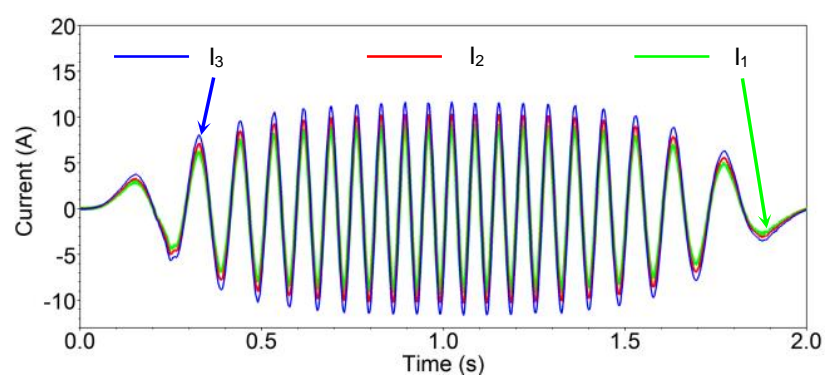

Fig. 9. Different armature current waveforms with different limiting values.

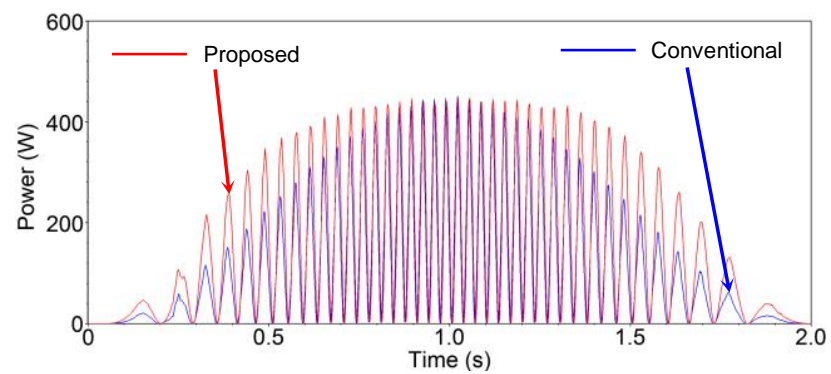

Fig. 10. Electrical power output with and without using the proposed method.

Figs. 11-14 show the PMLG's magnetic properties using this proposed method. Fig. 11 shows the magnetic flux density of the PMLG when the translator has the highest velocity with respect to the stator when aligning with the translator core during the simulation period. The air gap between the stator and the translator is maintained to the heiger value so that the coil current is kept within a spacified value. Fig. 12 shows the magnetic flux density of the PMLG when the translator has the lowest velocity with respect to the stator aligning with the last segment of the translator core. The air gap is then maintained to a lower value so that the coil current is boosted up, maintaining the current limit.

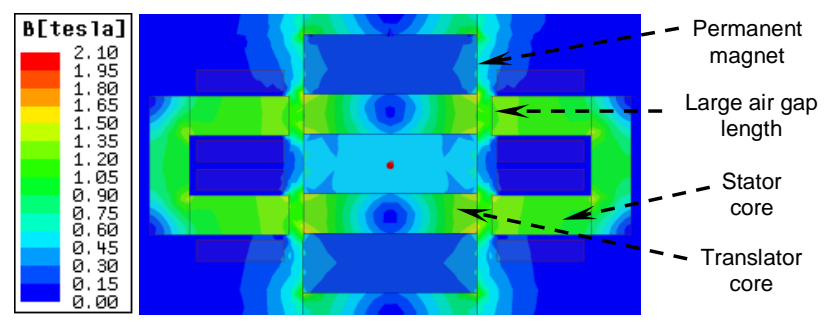

Fig. 11. Magnetic flux density of the PMLG for maximum air gap. 


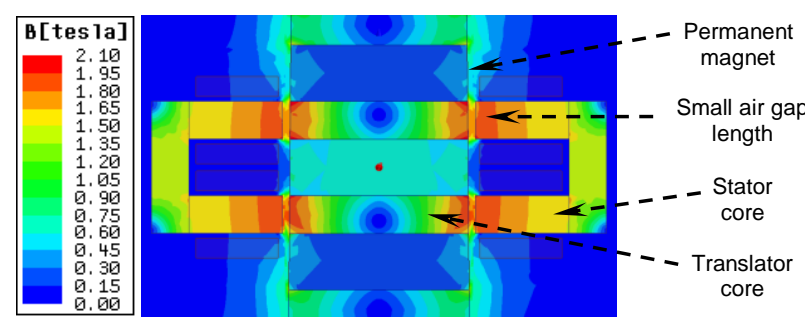

Fig. 12. Magnetic flux density of the PMLG for minimum air gap.

Different parameters of the PMLG are tabulated in Table I. Figs. 13 and 14 represent the magnetic field intensity, $H$ of the PMLG for the maximum and minimum air gap lengths when the velocities of the translator reaches its highest and lowest value, respectively at the time of the vertical alignments between the stator and the translator cores.

TABLE I

DIMENSIONS OF THE PMLG

\begin{tabular}{lc}
\hline \hline Name of the items & Value \\
\hline Air gap length between the stator and translator $(\mathrm{mm})$ & $3 \sim 6$ \\
Thickness of the stator pole $(\mathrm{cm})$ & 1.6 \\
Width of the stator pole shoe $(\mathrm{cm})$ & 4 \\
Thickness of the permanent magnet $(\mathrm{cm})$ & 2.4 \\
Vertical distance of the stator poles $(\mathrm{cm})$ & 4 \\
Thickness of the translator core $(\mathrm{cm})$ & 1.6 \\
Vertical distance of the translator for one cycle $(\mathrm{cm})$ & 8 \\
Height of the stator pole shoe $(\mathrm{cm})$ & 1.6 \\
Length of the cross section of the copper coil $(\mathrm{cm})$ & 3.5 \\
Width of the cross section of the copper coil $(\mathrm{mm})$ & 8.5 \\
Total width of the stator poles $(\mathrm{cm})$ & 5.6 \\
Stroke length of the translator $(\mathrm{m})$ & 1.6 \\
Time period of the oceanic wave $(\mathrm{s})$ & 4 \\
Velocity of the translator $(\mathrm{m} / \mathrm{s})$ & $0 \sim 1.25$ \\
\hline \hline
\end{tabular}

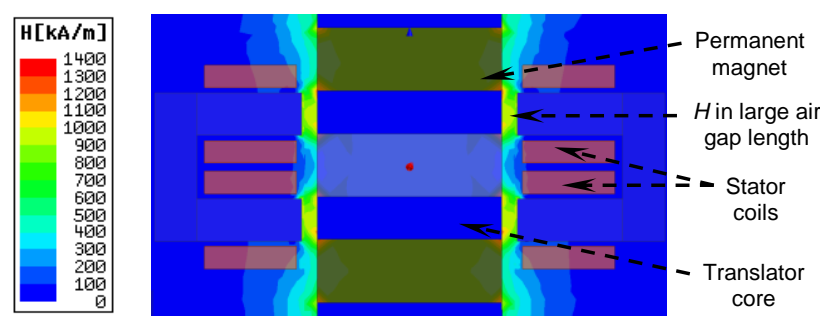

Fig. 13. Magnetic field intensity of the PMLG for the maximum air gap.

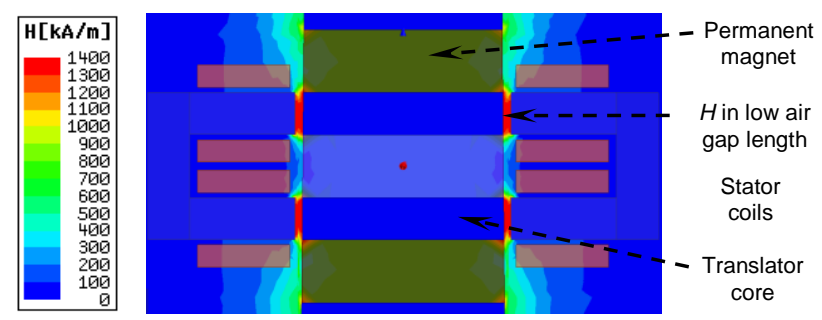

Fig. 14. Magnetic field intensity of the PMLG for the minimum air gap.

\section{CONCLUSION}

From the simulation results and analyses, it is found that the proposed method has effectively solved the demagnetization problem. The additional advantage of this method is that the average electrical power generation of the PMLG with variable air gap is increased, compared to that of the fixed air gap length for the same PMLG.
With the same current limit and operating point in the demagnetization curve, the electrical power generation of the PMLG is $161.23 \mathrm{~W}$ for the variable air gap. On the other hand, the generated electrical power is $199.566 \mathrm{~W}$ of the same PMLG for the proposed variable air gap method. Therefore, $23.77 \%$ more electrical power generation is achieved in the same PMLG by prevention of demagnetization. The proposed method is also applicable to oher PMLGs.

\section{REFERENCES}

[1] A. R. A. Arkadan, N. Al-Aawar, and A. O. Hariri, "EMTaguchi module for characterization of WAD," IEEE Trans. Magn., vol. 51, no. 3, article xxx, March 2015.

[2] A. Clément, et al., "Wave energy in Europe: Current status and per- spectives," Renew. Sustain. Energy Rev., vol. 6, no. 5, pp. 405-431, Oct. 2002.

[3] H. Polinder, J. G. Slootweg, M. J. Hoeijmakers, and J. C. Compter, "Modeling of a linear PM machine including magnetic saturation and end effects: maximum force-tocurrent ratio," IEEE Trans. Ind. Appl., vol. 39 pp. 16811688, 2003.

[4] N. Bianchi and S. Bolognani, "Design techniques for reducing the cogging torque in surface-mounted PM motors," IEEE Trans. Ind. Appl., vol. 38, no. 5, pp. 12591265, 2002.

[5] L. Dosiek and P. Pillay, "Cogging torque reduction in permanent magnet machines," IEEE Trans. Ind. Appl., vol. 43, no. 6, pp. 1565-1571, Nov./Dec. 2007.

[6] J. X. Jin, L. H. Zheng, Y. G. Guo, and J. G. Zhu, "Performance characteristics of an HTS linear synchronous motor with HTS bulk magnet secondary," IEEE Trans. Ind. Appl., vol. 47, no. 6, pp. 2469-2477, Nov./Dec.2011.

[7] J. Prudell, M. Stoddard, E. Amon, T. K. A. Brekken, and A. V. Jouanne, "A permanent-magnet tubular linear generator for ocean wave energy conversion," IEEE Trans. Ind. Appl., vol. 46, no. 6, pp. 2392-2400, Nov./Dec. 2010.

[8] L. Cappelli, F. Marignetti, G. Mattiazzo, E. Giorcelli, G. Bracco, S. Carbone, and C. Attaianese, "Linear tubular permanent-magnet generators for the inertial sea wave energy converter," IEEE Trans. Ind. Appl., vol. 50, no. 3, pp. 1817-1828, May/Jun. 2014.

[9] R. Vermaak and M. J. Kamper, "Experimental evaluation and predictive control of an air-cored linear generator for direct-drive wave energy converters," IEEE Trans. Ind. Appl., vol. 48, no. 6, pp. 1817-1826, Nov./Dec. 2012.

[10] S. T. Boroujeni, J. Milimonfared, and M. Ashabani, "Design, prototy-ping, and analysis of a novel tubular permanent-magnet linear machine," IEEE Trans. Magn., vol. 45, no. 12, pp. 5405-5413, Dec. 2009.

[11] L. Huang, H. Yu, M. Hu, J. Zhao, and Z. Cheng, "A novel flux-switching permanent-magnet linear generator for wave energy extraction application," IEEE Trans. Magn., vol. 47, no. 5, pp. 1034-1037, May 2011.

[12] J. A. García-Alzórriz, J. Grau, R. Córdoba, and J. Muela, "A novel double-sided flat rectangular linear permanent magnets synchronous generator for sea wave energy application," in Proc. the 7th Int. Conf. on Electrical and Electronics Engineering, Bursa, 2011, pp. I-248-252.

[13] O. Farrok and M. M. Ali, "A new technique to improve the linear generator designed for oceanic wave energy conversion," in Proc. Int. Conf. on Electrical and Computer Engineering, Dhaka, Bangladesh, Dec. 20-22, 2014, pp. 714-717.

[14] O. Farrok, M. R. Islam, and M. R. I. Sheikh, "Fuzzy logic 
based an improved controller for wave energy conversion systems," in International Conference on Electrical Engineering and Information Communication Technology, Dhaka, Bangladesh, May 21-23, 2015, pp. 1-6.

[15] O. Farrok, M. R. Islam, M. R. I. Sheikh, and W. Xu, “A new optimization methodology of the linear generator for wave energy conversion systems," in Proc. IEEE International Conference on Industrial Technology, Taipei, Taiwan, Mar. 14-17, 2016, pp. 1412-1417.

[16] O. Farrok, M. R. Islam, M. R. I. Sheikh, Y. G. Guo, J. G. $\mathrm{Zhu}$, and $\mathrm{W} . \mathrm{Xu}$, "Analysis and design of a novel linear generator for harvesting oceanic wave energy," Proc. IEEE Int. Conf. Appl. Supercond. Electromagn. Devices, Shanghai, China, Nov. 20-23, 2015, pp. 272-273.

[17] O. Farrok, M. R. Islam, M. R. I. Sheikh, Y. G. Guo, J. G. $\mathrm{Zhu}$, and W. Xu, "A novel superconducting magnet excited linear generator for wave energy conversion system," IEEE Trans. Appl. Supercond., vol. 26, no. 7, article 6207105, Oct. 2016.

[18] O. Farrok, M. R. Islam, and M. R. I. Sheikh, "Analysis of the oceanic wave dynamics for generation of electrical energy using a linear generator," J. Energy, vol. 2016, Article ID 3437027, 14 pages, 2016. 